\title{
Differential protein expression in Mycobacterium tuberculosis susceptible and multidrug resistant isolates
}

\author{
Hadizadeh Tasbiti $\mathrm{AR}^{1,4}$, Yari $\mathrm{Sh}^{1,4 \#}$, Ghanei $M^{1,2}$, Siadat $\mathrm{SD}^{1,4}$, Niknami $\mathrm{S}^{3}$, Bahrmand $\mathrm{A}^{1^{*}}$ \\ ${ }^{1}$ Tuberculosis Dept. Pasteur Institute of Iran. Tehran. Iran. \\ ${ }^{2}$ Chemical Injury Research Center, Baqiyatallah university of medical sciences, Tehran,Iran, \\ ${ }^{3}$ Department of health education, faculty of medical sciences, tarbiat modares university, Tehran, Iran. \\ ${ }^{4}$ Microbiology Research Center (MRC), Pasteur Institute of Iran. Tehran. Iran .
}

\begin{abstract}
Introduction: Infections by multidrug resistant Mycobacterium tuberculosis (MDR-TB) is a major public health challenge. Secretion of proteins by M. tuberculosis plays an important role in the pathogenesis of the bacterium. We compared the protein profiles of susceptible $M$. tuberculosis and MDR-TB isolates using proteomic analyses, namely two dimensional gel electrophoresis (2DE) and mass spectrometry (MS). Methods: The bacilli were cultured on Middlebrook $7 \mathrm{H} 9$ medium and bacterial colonies were mechanically disrupted and proteins were extracted by ammonium sulfate. The 2DE and MS analyses were performed using Ettan IP Gphor 3 isoelecteric system and Autoflex II TOF/TOF, respectively. Results: Our study showed that in comparison to the sensitive strains, 27 proteins were over-expressed in the MDR isolates and these proteins were mainly involved in the cellular metabolism, cell wall and membrane structures and bacterial respiration. Bactoferritin (Rv1876) has been shown to play a role in antibacterial resistance. Increased intensity of Rv2031c, a heat shock protein (Alpha-crystallin/HspX), was also observed in the whole cell lysate of the MDR-TB. This protein is a marker of the latent TB and has been proposed as a target for vaccine development. Conclusion: Our results identified proteins that are overexpressed in the resistant M. tuberculosis which could be used as antibacterial targets or vaccine candidates.
\end{abstract}

KEYWORDS: Multidrug resistant Mycobacterium tuberculosis (MDR-TB), tuberculosis proteins, protein candidate, Mycobacteria antigen.

\section{INTRODUCTION}

Tuberculosis (TB) caused by Mycobacterium tuberculosis is present in all regions of the world as stated in WHO Global Tuberculosis Report 2014 which includes data compiled from 202 countries and territories [1]. This year's report shows even higher global totals for new TB cases and deaths in 2013 than previous years, reflecting the increased and improved use of national data in this regard. As parts of the post 2015 global TB strategy, the early diagnosis is emphasized and the inclusion of drug susceptibility testing (DST) is considered as a universal standard for patient care for both the new and the previouslytreated patients. Detection of TB without investigation for drug resistance can lead to ineffective treatments.

Inappropriate treatment regimens and poor patient compliance

\footnotetext{
*Corresponding Author: Ahmadreza Bahrmand, Tuberclulosis Department, Pasteur Institute of Iran, Tehran, Iran.

Email: padideh79@yahoo.com

Tel/Fax: (+98) 2166968853/ (+98) 2166465132

\#Co-Corresponding Author: Shamsi Yari, TB Protein Chemistry Lab,

Tuberculosis dept, Pasteur Institute of Iran, Tehran, Iran.

Email: tbchemistry@yahoo.com

Tel/Fax: (+98) 2166968853/ (+98) 2166465132
}

have led to the appearance of multi drug resistant (MDR) and extensively drug resistant (XDR) M. tuberculosis strains which make the treatment of TB costly, lengthy and difficult [2]. By definition, Multidrug resistant Mycobacterium tuberculosis (MDR-TB), is resistant to at least isoniazid and rifampin. The treatment of infections caused by MDR-TB is a major concern for TB control programs worldwide because it requires prolonged use of multiple second-line anti-TB drugs which are more expensive and toxic than the first-line drugs. TB death toll is very high although it could be prevented with a timely diagnosis and correct treatments. Over $95 \%$ of TB deaths occur in low- and middle-income countries where it is among the top three causes of mortality for women aged 15 to 44 . The rate of new cases has been falling worldwide for about a decade and there has been a 45\% reduction in TB mortality rate since 1990 . Moreover, 56 million people have been successfully treated between 1995 and 2012 [1]. Therefore, rapid detection of drug resistance and therapeutic options according to the resistance profiles are important elements for controlling MTB infections. In the present study, two dimensional gel electrophoresis (2DE) and mass spectrometry (MS) along with relative protein expression abundance calculations were used to compare the 
membrane protein expression profiles of susceptible and MDRTB strains. The recent discovery of a true outer membrane in the cell wall of MTB has unraveled the previously-known hostbacterial interactions and consequently has made the antimicrobial susceptibility more complicated [3]. The aim of this study was to find proteins that could further explain the different phenotypes of the two strains, especially their distinct abilities which could be used to develop new drugs against MTB.

\section{MATERIALS and METHODS}

\section{Bacterial strains}

Sensitive MTB-1126 and MDR-TB isolates were obtained from TB-Bank of Pasteur Institute of Iran and were cultured on Middlebrook $7 \mathrm{H} 9$ medium at $37^{\circ} \mathrm{C}$ for 4 weeks. MDR-TB was defined as TB caused by strains resistant to at least isoniazid and rifampicin (Sigma, USA) and was determined by minimum concentration method (MIC) at a concentration of $0.2,40,2.0$, and $4.0 \mu \mathrm{g} / \mathrm{ml}[4-6]$.

\section{Sample preparation}

The bacterial cells were gathered by centrifugation at $5000 \mathrm{rpm}$ $\left(45 \mathrm{~min}\right.$ at $4^{\circ} \mathrm{C}$ ) and the pellets were washed with Tris $50 \mathrm{mM}$ twice and were resuspended in $50 \mathrm{mM}$ Tris, $150 \mathrm{mM} \mathrm{NaCl}, 1$ $\mu \mathrm{g} / \mathrm{ml}$ DNase, $1 \mathrm{mM}$ PMSF, $20 \mathrm{mM}$ sucrose, $10 \mathrm{mM} \mathrm{MgCl} 2$, $0.02 \mathrm{~g}$ sodium azide, $10 \%$ glycerol $(\mathrm{pH} \sim 8.0)$. The pellets were sonicated for $1 \mathrm{~h}$ at $50 \mathrm{~Hz}$ (Bandelin, GM -220 Germany). The unbroken cell debris was put off by centrifugation at $5000 \mathrm{rpm}$ $\left(45 \mathrm{~min}\right.$ at $\left.4{ }^{\circ} \mathrm{C}\right)$. The proteins were precipitated by ammonium sulfate and incubated overnight at $4^{\circ} \mathrm{C}$. After centrifugation at $13000 \mathrm{rpm}$ for $45 \mathrm{~min}$ at $-4^{\circ} \mathrm{C}$, the proteins were suspended in $50 \mathrm{mM}$ Tris $(\mathrm{pH} \sim 8)$ and dialyzed exhaustively against $50 \mathrm{mM}$ Tris (pH 8). The amounts of proteins were quantified using Bradford assay [7].

2DE

2DE was performed using the Ettan IPGphor 3 isoelecteric focusing system (GE Healthcare, Sweden). The gel strips ( $\mathrm{pH}$ 4-7 and length $11 \mathrm{~cm}$ ) were focused on an IEF unit according to the following program: $500 \mathrm{~V}$ for $1 \mathrm{~h}, 1000 \mathrm{~V}$ for $1 \mathrm{~h}, 8000 \mathrm{~V}$ for $3 \mathrm{~h}$, finally $8000 \mathrm{~V}$ for $20 \mathrm{KVh}$, at $20^{\circ} \mathrm{C}$ and a current limit of $50 \mu \mathrm{A}$ per strip. After IEF, the strips were equilibrated in equilibration buffer (15 min in 6M urea), 20\% SDS, $1.5 \mathrm{M}$ Tris, $50 \%$ glycerol, $2 \%$ DTT, $2.5 \%$ idoacetamide ( $\mathrm{pH} \sim 8.8$ ). The proteins were separated in second dimension on $12 \%$ SDSPAGE in a vertical electrophoretic dual gel and were visualized by Coomassie (R-250) staining method [8-9].

\section{MS and data analyses}

Excised protein bands $(1 \times 1 \mathrm{~mm})$ were sent to the Chemistry Department, York University, UK for MS. The purified peptides were applied to an Anchor Chip and were analyzed by MALDI-TOF Mass Analyzer (Bruker Daltronic Reflex III). Aliquot of each peptide mixture was carried out a ground steel MALDI (target plate). Fragmentation was committed in LIFT mode in absent of a collision gas. The primary calibration was performed for MS/MS spectra, which were subtracted and smoothed (cycles 4, width $0.15 \mathrm{~m} / \mathrm{z}$, Savitsky-Golay). For detection of monoisotopic peak, a SNAP averaging algorithm was used with a minimum S/N of 6 . Analysis software (Bruker flex, version 3.3) was performed to spectral processing and peak list generation. By locally-running copy of the Mascot program (Matrix Science Ltd., version 2.4) through the Bruker Protein scape interface (version 2.1), the Tandem mass spectral data were submitted to database searching engine. The results were purified to accept peptides with just score of 0.05 or lower, allowing to acquire higher confidence identifications including Tandem spectral data in MASCOT $<\mathrm{http}: / / \mathrm{www}$.matrixscience.com/> searches and the data were assimilated with the MASCOT database for any sequence matches[10].

\section{RESULTS}

Our study identified 27 proteins that were upregulated or overexpressed in the MDR-TB strains compared to the sensitive isolates as summarized in Table 1. All the detected spots in the Coomassie Brilliant Blue stained 2DE gels were cut and applied for in gel digestion procedures, followed by MALDI-FOF/MS analysis. The analysis of two independent strains (sensitive and MDR-TB isolates), using image master, Melanie analysis software (version 6.0) revealed that the majority of these proteins were common to both strains. However, 27 different proteins were differentially expressed (upregulated/expressed proteins) in MDR compared to the sensitive isolates. The majority of the spots were found in the $\mathrm{pH}$ range of 4.0 to 6.5 with molecular weights ranging from 14.4 to more than $45.0 \mathrm{kDa}$. The identified proteins were short chain dehydrogenase/reductase (Rv3057c), peptidyl-prolyl-cistrans (Rv0009), putative dioxygenase (Rv3161c), pre-protein translocase subunit SEcE2 (Rv0379), 2 cell wall proteins (Rv0379, Rv3614c), 3 information pathways proteins (Rv0009, Rv0685, Rv2986c), 4 hypothetical proteins (Rv0443, Rv2114, Rv3311 and Rv0831), 2 secreted proteins (Rv3804c and Rv3614c), 2 heat shock proteins (Rv3418c and Rv2031c) and 14 proteins belonging to metabolism and respiration pathways. The Functions of identified MDR-TB proteins on the 2DE gels followed by analysis with MALDI-TOF MS are shown in Fig. 1. Fourteen proteins of intracellular MDR isolate (40.5\%) belonged to the intermediary metabolism and the respiration category. Five proteins of the intracellular MDR isolate $(16.7 \%)$ were conserved hypothetical proteins which were functionally characterized by the bioinformatics tools.

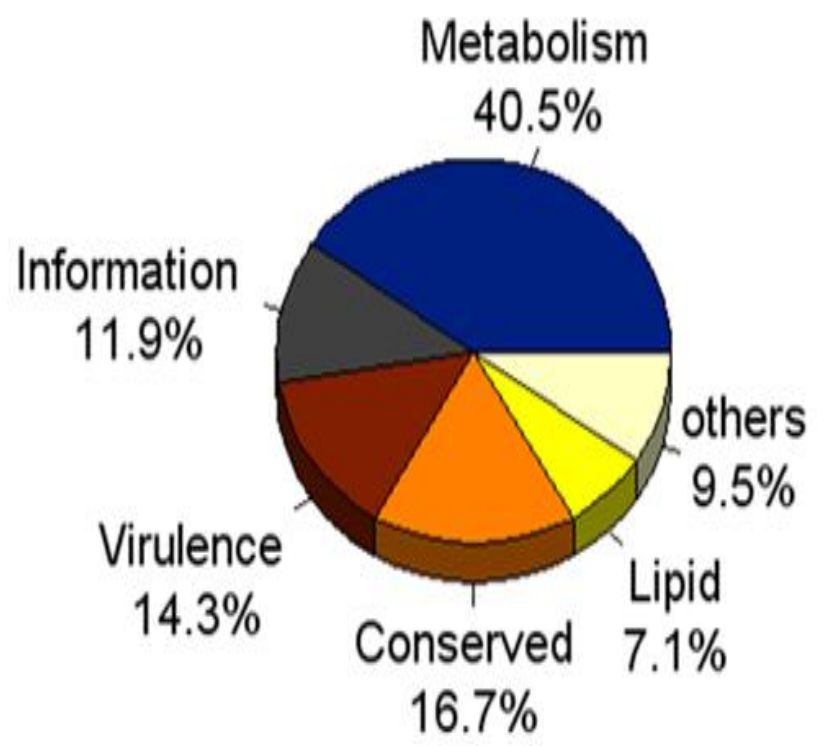

Fig. 1. Function of identified MDR-TB proteins on the 2DE gels followed by analysis with MALD-TOF MS. 
Table 1. Identified expressed proteins of MDR Tuberculosis isolates on the 2DE gels and MALDI-TOF mass spectrometry.

\begin{tabular}{|c|c|c|c|c|c|c|c|c|c|}
\hline No & Gene & Gene product & (MW) & (PI) & Protein length (aa) & Location (kb) & Score & Gene length (bp) & Putative function \\
\hline 1 & Rv3418C & $10 \mathrm{kDa}$ chaperonin GroES & 10.79 & 4.51 & 100 & 3836.99 & 470 & 303 & Virulence, detoxification \\
\hline 2 & Rv2971 & oxidoreductase & 30.51 & 4.70 & 282 & 3326.1 & 95 & 849 & metabolism and respiration \\
\hline 3 & Rv1308 & ATP synthase alpha chain AtpA & 59.48 & 5.03 & 549 & 1463.23 & 332 & 1650 & metabolism and respiration \\
\hline 4 & Rv0831C & Conserved protein & 30.17 & 5.09 & 271 & 922.894 & 226 & 816 & conserved hypothetical \\
\hline 5 & Rv1876 & Bacterioferritin & 18.44 & 4.51 & 159 & 2125.34 & 220 & 480 & metabolism and respiration \\
\hline 6 & Rv0379 & pre-protein translocase subunit SEcE2 & 16.68 & 6.98 & 135 & 455.977 & 136 & 216 & cell wall cell processes \\
\hline 7 & Rv1240 & Malate dehydrogenase & 34.35 & 4.65 & 329 & 1383.21 & 210 & 990 & metabolism and respiration \\
\hline 8 & Rv2114 & Conserved protein & 28.26 & 4.8 & 260 & 2373.83 & 254 & 624 & conserved hypothetical \\
\hline 9 & Rv0443 & Conserved protein & 13.27 & 4.60 & 128 & 532.396 & 173 & 516 & conserved hypothetical \\
\hline 10 & Rv3248C & Adenosylhomocysteinase & 54.4 & 5.07 & 495 & 3628.16 & 86 & 1488 & metabolism and respiration \\
\hline 11 & Rv3804c & secreted antigen Ag85B & 34.72 & 6.82 & 495 & 4265.64 & 120 & 1017 & lipid metabolism \\
\hline 12 & Rv2986C & $35 \mathrm{kDa}$ protein $\mathrm{OS}$ & 29.24 & 5.71 & 270 & 3343.18 & 84 & 645 & Information pathways \\
\hline 13 & Rv3614c & ESX-1secretion-associated protein & 19.87 & 3.93 & 184 & 4054.14 & 306 & 555 & cell wall cell processes \\
\hline 14 & Rv2215 & dihydrolipoamide actyltransfrase & 57.11 & 4.9 & 553 & 2481.97 & 181 & 1662 & metabolism and respiration \\
\hline 15 & Rv1392 & S-adenosylmethionine synthetase & 43.13 & 4.95 & 403 & 1566.82 & 238 & 1212 & metabolism and respiration \\
\hline 16 & Rv3028C & electron transfer flavoprotein subunit alpha & 31.67 & 4.71 & 318 & 3387.07 & 414 & 957 & metabolism and respiration \\
\hline 17 & Rv1189 & RNA polymerase SigI & 24.72 & 5.39 & 226 & 1332.09 & 76 & 873 & metabolism and respiration \\
\hline 18 & Rv3311 & Conserved protein & 45.81 & 4.14 & 420 & 3698.12 & 142 & 1263 & conserved hypothetical \\
\hline 19 & Rv3057c & short chain dehydrogenase/reductase & 30.67 & 5.27 & 293 & 3417.8 & 344 & 864 & metabolism and respiration \\
\hline 20 & Rv0462 & Dihydrolipoyl dehydrogenase OS & 49.43 & 5.53 & 464 & 552.614 & 589 & 1395 & metabolism and respiration \\
\hline 21 & Rv2031c & Heat shock protein HspX & 16.22 & 4.75 & 144 & 2278.5 & 76 & 435 & virulence, detoxification, \\
\hline 22 & Rv0685 & Elongation factor $\mathrm{Tu}$ & 43.56 & 5.2 & 396 & 784.821 & 302 & 1191 & information pathways \\
\hline 23 & Rv3161c & Possible dioxygenase & 42.50 & 5.00 & 382 & 3529.99 & 213 & 1149 & metabolism and respiration \\
\hline 24 & Rv0462 & Dihydrolipoyl dehydrogenase OS & 49.43 & 5.53 & 464 & 552.614 & 589 & 1395 & metabolism, and respiration \\
\hline 25 & Rv0009 & Peptidyl-prolyl-cis-trans & 19.23 & 6.2 & 182 & 12.468 & 198 & 549 & information pathways \\
\hline 26 & Rv3699 & Conserved protein & 25.04 & 4.69 & 233 & 4142.04 & 287 & 702 & conserved hypothetical \\
\hline 27 & Rv1392 & S-adenosylmethionine synthetase & 43.13 & 4.95 & 403 & 1566.82 & 238 & 1212 & metabolism and respiration \\
\hline
\end{tabular}




\section{DISCUSSION}

Our study of the protein profiles of MDR and the sensitive $M$. tuberculosis isolates showed that the majority of the proteins in the whole cell lysates were present in both groups and had similar relative abundance. However, the overexpressed protein in the $\mathrm{pH}$ range in this study were antigen 85 (Ag85), bacterioferitin, pre protein SEcE2, EsX-1 secretion associated protein, RNA polymerase SigI, short chain dehydrogenase/reductase, heat shock protein HspX, putative dioxygenase and peptidyl polyl cis-trans. Ag85 protein is responsible for the high tendency of mycobacteria for fibronectin, the great adhesive glycoprotein which facilitates the attachment of MTB to murine alveolar macrophages. The protein also helps to maintain the integrity of the cell wall by catalyzing the convey of mycolic acids to the cell wall's arabinogalactan and through the synthesis of cord factor [11]. Ag85A and Ag85B (Rv3804c) have been reported to be ideal vaccine candidates in a number of studies [2]. Bacterioferritin, bfrA, is involved in iron storage which is a necessary requirement for the growth of bacilli. However, its excess as a source of free iron is toxic and leads to the production of reactive oxygen species and consequently oxidative damage. Ferritin has been reported to show increased intensity under the absence of oxygen condition and nitric oxide deficiency but such activity has not been observed for bacterioferritin. The function of bacterioferritins may not be just limited to the iron uptake and they may be contributing to other metabolic activities which their mechanisms are still unclear [12]. These results showed the upregulation of bacterioferritin (Rv1876) in MDR-TB isolates. It is suggested that the exclusive expression of bacterioferritin in MDR-TB indicates its probable involvement in resistance to the first line of anti-mycobacterial drugs.

Although our findings show a significant improvement compared to these studies, there are some differences between the present and the earlier studies. The discrepancy between the observed and the predicted protein $\mathrm{pI}$ values is a common feature of proteomic analyses using 2DE gels. This is considered, amongst other things, to be due to the conformational differences, post-translational modifications and other processing events of proteins which may affect their migration. MS identification of mycobacterial proteins from 2D gels has only been applied so far to in vitro grown cultures where abundant amounts of proteins are available for analysis.

Peptidyl-prolyl cis-trans isomerase (Rv0009) also known as cyclophilin is iron-regulated and is necessary for protein folding and is thought to participate in processes such as signaling, cell surface recognition and chaperoning. Rv0009 is a major cellular target for the immunosuppressive drug cyclosporine [13]. Calcium dodecin (Rv0379) binds to calcium ions and plays a role in sequestering additional small ligands and is involved in protein transport [4].

Arockiasamy et al. have shown that the earliest interactions of MTB with macrophages result in a number of alterations in $\mathrm{Ca}+2$ signaling events that are critical for the phagosomes maturation [14]. Malate dehydrogenase, (Rv1240) has been identified in the immunodominant membrane fractions or cell wall of MTB [15]. The elongation factor (EF-Tu) was identified at another spot with significantly different pI and higher molecular mass, suggesting that it was a proteolytic degradation product. The increase in EF-Tu expression may reflect either an overall increase in the protein synthesis related to the growth in the intracellular environment or may indicate some regulatory functions during the translational control of gene expression. A previous study by Wong et al. has demonstrated that EF-Tu is up-regulated when MTB is exposed to high iron conditions in vitro. EF-Tu (Rv0685) belongs to GTP-binding elongation factor family and has been predicted as a possible vaccine candidate [16].

Two of the upregulated proteins in this study were identified as molecular chaperones. These are a diverse set of proteins that mediate the correct folding, assembly, transport and degradation of other proteins and are induced under oxygen deficient conditions [17]. These proteins are considered as important players in the survival of mycobacteria in the presence of drugs and elucidation of their exact contribution to the bacterial drug resistance may help to develop new antimycobacterial therapeutics. In conclusion, here we focused on comprehensive comparison of proteins from sensitive and MDR-TB strains based on the high resolution 2DE patterns. This comparison is primarily aimed at the identification of proteins present in MDR-TB, but absent in susceptible $M$. tuberculosis species, are considered as valuable antigens for novel diagnostic, therapeutic and/or vaccination strategies against TB.

\section{ACKNOWLEDGEMENT}

We are grateful to the members of the Tuberculosis Department of Pasteur Institute of Iran for the collection of TB strains. This study was funded by Pasteur Institute of Iran.

\section{CONFLICT OF INTEREST}

The authors declare that they have no conflict of interest.

\section{REFERENCES}

1. Organization WH. World Health Organization global tuberculosis report. Online availanle ht: h:/Www int/tb/publications/global_report/gtbr12_main pdf. 2013.

2. Singhal N, Sharma P, Kumar M, Joshi B, Bisht D. Analysis of intracellular expressed proteins of Mycobacterium tuberculosis clinical isolates. Proteome Sci. 2012;10(1):14. doi:10.1186/1477-5956-10-14.

3. Malen H, De Souza GA, Pathak S, Softeland T, Wiker HG. Comparison of membrane proteins of Mycobacterium tuberculosis H37Rv and H37Ra strains. BMC Microbiol. 2011;11:18. doi:10.1186/1471-2180-11-18.

4. Malen H, Pathak S, Softeland T, de Souza GA, Wiker HG. Definition of novel cell envelope associated proteins in Triton X-114 extracts of Mycobacterium tuberculosis H37Rv. BMC Microbiol. 2010;10:132. doi:10.1186/1471-2180-10-132.

5. Kent PT, Kubica GP. Public health mycobacteriology: a guide for the level III laboratory. US Department of Health and Human Services, Public Health Service, Centers for Disease Control; 1985.

6. Organization WH. Guidelines for drug susceptibility testing for secondline anti-tuberculosis drugs for DOTS-plus. 2001.

7. Bradford MM. A rapid and sensitive method for the quantitation of microgram quantities of protein utilizing the principle of protein-dye binding. Anal Biochem. 1976;72:248-54.

8. Gu S, Chen J, Dobos KM, Bradbury EM, Belisle JT, Chen X. Comprehensive proteomic profiling of the membrane constituents of a Mycobacterium tuberculosis strain. Molecular \& Cellular Proteomics. 2003;2(12):1284-96.

9. GE Healthcares. 2DE Electrophoresis. Principles and methods 18-102218. Bio sciences, Brokgatan.30 Uppsala.Sweden 2004.http://www.gelifesciences.com.

10. Shevchenko A, Tomas H, Havlis J, Olsen JV, Mann M. In-gel digestion for mass spectrometric characterization of proteins and proteomes. Nat Protoc. 2006;1(6):2856-60. doi:10.1038/nprot.2006.468.

11. Zvi A, Ariel N, Fulkerson J, Sadoff JC, Shafferman A. Whole genome 
identification of Mycobacterium tuberculosis vaccine candidates by comprehensive data mining and bioinformatic analyses. BMC Med Genomics. 2008;1:18. doi:10.1186/1755-8794-1-18.

12. Kumar B, Sharma D, Sharma P, Katoch VM, Venkatesan K, Bisht D. Proteomic analysis of Mycobacterium tuberculosis isolates resistant to kanamycin and amikacin. Journal of proteomics. 2013;94:68-77.

13. Fleischmann RD, Alland D, Eisen JA, Carpenter L, White O, Peterson J et al. Whole-genome comparison of Mycobacterium tuberculosis clinical and laboratory strains. J Bacteriol. 2002;184(19):5479-90.

14. Arockiasamy A, Aggarwal A, Savva CG, Holzenburg A, Sacchettini JC. Crystal structure of calcium dodecin (Rv0379), from Mycobacterium tuberculosis with a unique calcium-binding site. Protein Sci. 2011;20(5):827-33. doi:10.1002/pro.607.
15. Mattow J, Siejak F, Hagens K, Becher D, Albrecht D, Krah A et al Proteins unique to intraphagosomally grown Mycobacterium tuberculosis Proteomics. 2006;6(8):2485-94. doi:10.1002/pmic.200500547.

16. Monahan IM, Betts J, Banerjee DK, Butcher PD. Differential expression of mycobacterial proteins following phagocytosis by macrophages. $\quad$ Microbiology. 2001;147(Pt 2):459-71. doi:10.1099/00221287-147-2-459.

17. Sharma P, Kumar B, Singhal N, Katoch VM, Venkatesan K, Chauhan DS et al. Streptomycin induced protein expression analysis in Mycobacterium tuberculosis by two-dimensional gel electrophoresis \& mass spectrometry. Indian J Med Res. 2010;132:400-8. 